\title{
21
}

\section{How teaching through IT modifies the teaching curriculum}

\author{
G. Rawlings \\ University of Central Lancashire, \\ Preston, Lancashire. Great Britain. Tel 01772893664 \\ E-Mail G.Rawlings@UCLAN.AC.UK.
}

\begin{abstract}
This paper seeks to compare traditional delivery of the Business and Management subjects with that of an approach that seeks to teach through IT. To achieve this objective this paper will take a traditionally taught area of Business and Management education and compare the traditional "Business Textbook" approach to the subject with a teaching approach that develops the subject through the use of IT.
\end{abstract}

\section{Keywords}

Business, management, traditional teaching, teaching through IT

\section{INTRODUCTION}

This paper will focus on the caparison of teaching in the quantitative areas, in particular focus on introductory statistics courses as taught to undergraduates in Business and Management Faculties.

Teaching through IT means that the subject material delivered has to be reviewed and re-evaluated, and the traditional learning objectives questioned and modified. This leads to the presentation of a course that contains relatively similar materials, but with considerable differences in the emphasis and delivery of the topics comprising the course.

The lessons learned and an indication of the future developments will be discussed.

\section{THE TRADITIONAL APPROACH}

\subsection{Textbook Approaches}

Examination of a range of textbooks aimed at students taking an introductory courses in statistics for business shows a relatively common approach. This approach is characterised by the following broad structure: 
The early chapters give an introduction to what the study of statistics is, followed by a discussion of the basic principles of the graphical presentation of sample data and descriptive statistics.

The emphasis then changes to a discussion of the ideas of probability theory, leading to the ideas of probability distributions and a random variable leading on to sampling distributions. Following this block of theory the ideas of estimation and hypothesis testing are introduced.

The later chapters then cover the analysis of variance, regression modelling followed by a range of issues typically time-series and forecasting, decision theory non-parametric statistics and quality control.

\subsection{Traditional teaching approaches}

Here the evidence is more anecdotal based on experiences as an advisor, external examiner and discussion with colleagues at various institutions. Experience generally suggests that the textbook framework is followed in the sense that a common model is the start with ideas of data collected from samples and how to summarise and present basic descriptive and summary information. Then switch to probability theory leading to a discussion of distribution theory, in particular the normal distribution then discuss sampling distributions and hypothesis testing. Regression modelling and other topics such as forecasting, quality control then typically follow.

\subsection{Critique of the traditional approach}

Experience suggests that the major problems that business students studying a course in introductory statistics comprehending the role of the theory as developed based on the ideas of probability theory and its use in the analysis of sample data. The better student can certainly follow the operational procedures developed in terms of manipulating the theoretical structures developed in the course but questioning about the subtle issues such as what is role of the ideas of a population and population parameters relative to sample parameters generally reveals some confusion. This is not surprising given the mathematical background of a typical student in a Business Faculty and the ideas and issues of handling the ideas of probability and statistical distributions require a reasonable level of mathematical skills.

On completion of a traditional introductory course in business statistics what will the student be able to do? Could they handle the analysis of a data set, derived for example from a survey, a common business activity? In general issues of data analysis are not explicitly developed although the key ideas are developed in the traditional approach.

\section{TEACHING THROUGH IT}

\subsection{Objectives}

The initial objectives were to provide an introductory course in Business Statistics that would provide a sufficient framework for the student to be able to make a reasonable attempt at analysing a set of sample data. The course should provide a framework for analysis that would show the need and role for coherent underlying theory to enable the analysis. The formal 
development of the underling theoretical structures can then be discussed within the context provided.

\subsection{Structure of the Course}

The starting point is similar to the traditional approach, a discussion of the need and use of statistics in business, this develops into a the discussion of a particular group of data sets, all of which are available in a format suitable for investigation using a statistical analysis package. (The package used being MINITAB).

Before a data set is provided the nature of the business problem under investigation is discussed, which is generally some market research type activity which is within the experience of the student group with the objective of :

- Showing that the business problem could be investigated by collecting some information establishing the need for a sample, and for it to be a fair and representative sample.

- Showing that one sample element will lead to the recording of a number of variables.

- The variables have different characteristics, at least one of the variables will directly relate to the objectives of the analysis, and is defined to be the response variable. The remaining variables which may then influence the response variable, are the factors.

A commonly used scenario is to investigate the factors that effect the amount spent in a supermarket. This requires a survey, the variable directly related to the objective of the analysis is "amount spent", the other variables being the factors that may or may not influence the amount spent.

Having defined this framework for the purpose of analysis, each variable can be classified as being either a measured variable (discrete or continuous) or an attribute variable. The objectives of the data analysis is to investigate examine the interaction between the response variable and each factor to see if the factor influences the response variable.

Four types of analysis according to the combination of variable types are possible these are summarised in the diagram below:

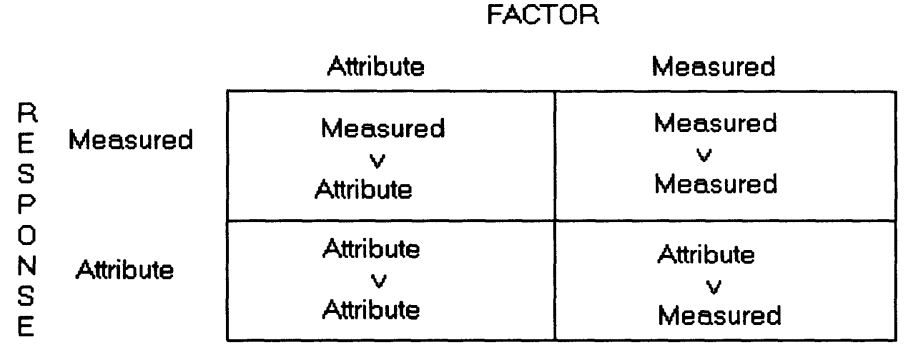

Figure 1

Each of these data analysis scenarios are discussed separately but within a consistent framework of the analytical methodology which as the first stage provides an initial data analysis method allowing a judgement to be made on one of three possible outcomes:

- There is no evidence of a relationship between the response and the factor,

- There is strong evidence of a relationship between the response and the factor. 
- The initial data analysis is inconclusive, hence further analysis is required, which will either point to there being no relationship or to there being a relationship, in which case the nature of the relationship is described.

This data analysis framework is shown in the diagram below:

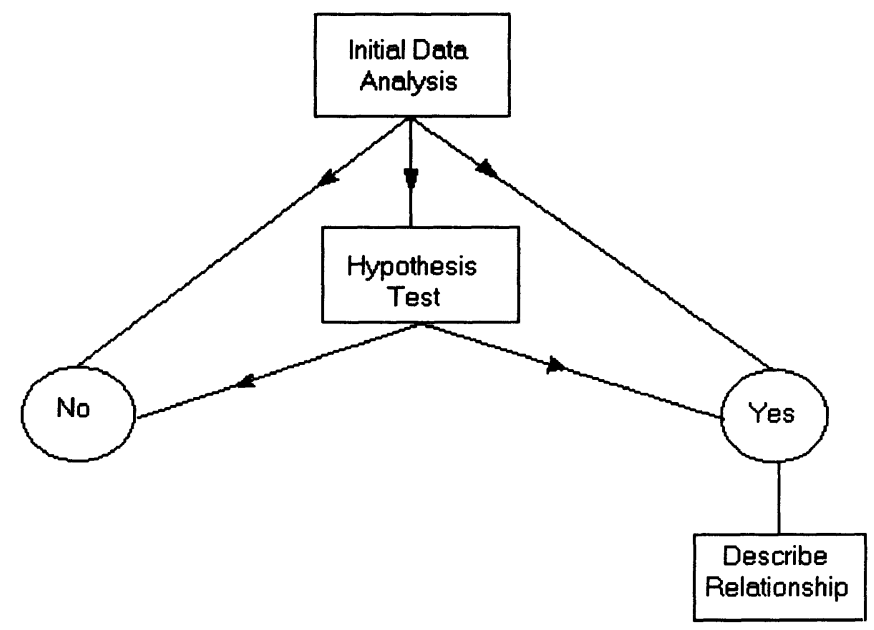

Figure 2

\subsection{The teaching approach}

For each combination of variable types, as illustrated in Figure 1, the teaching approach is common. Initially the population concepts are described in terms of having perfect information. Given perfect information the idea of a statistical distribution for both attribute and measured variables can be developed. For measured variables the usual descriptive statistics are introduced. The next step is to define what a relationship between the response and the factor is. With the exception of the measured response $v$ measured factor this is in terms of comparing the distributions and asking are the distributions the same for each attribute or do the distributions depend on the level of the attribute, is so then there is a relationship. The definition of a relationship when the two variables are measured is in terms of a conceptual visual plot of the two variables for the population and examining the patterns if any. Examples such as is the amount spent in a supermarket gender independent or gender dependent, does the amount spent in a supermarket depend on household income, and is class of degree gender independent or gender dependent provide useful illustrative examples.

The emphasis then switches to examining sample information, and a discussion of error due to sampling. Examining sample information means handling imperfect information, thus all statistical procedures need to be interpreted with this in mind. For each combination of variable types (Figure 1) the analysis is developed on the lines of illustrated in Figure 2. This diagram is a useful aid for the student. The Initial Data Analysis will either so a clear relationship, or no relationship or be inconclusive. If the Initial Data Analysis is inconclusive then a formal hypothesis test is required. The diagram aids the student in formally defining the hypotheses for 
the hypothesis test. The NO branch specifying the Null Hypothesis and the YES branch the Alternative Hypothesis. For example a measured response $v$ an attribute factor the NO branch, from the definition of a relationship means the population means for all the attributes are the same, the alternative, the YES branch indicating the population means are different.

The statistical package used is MINITAB and the commands introduced for the initial data analysis and hypothesis tests are given below:

\begin{tabular}{|c|c|c|}
\hline & \multicolumn{2}{|c|}{ FACTOR } \\
\hline & Attribute & Measured \\
\hline \multirow{3}{*}{ Measured } & $\begin{array}{l}\text { DESCRIBE / BY } \\
\text { BOXPLOT / BY }\end{array}$ & $\begin{array}{l}\text { PLOT } \\
\text { REGRESS }\end{array}$ \\
\hline & $\begin{array}{l}\text { TWOTEST } \\
\text { ONEWAY }\end{array}$ & $\begin{array}{l}\text { ANALYSIS OF VARIANCE } \\
\text { (from REGRESS) }\end{array}$ \\
\hline & TABLE & $\begin{array}{l}\text { DESCRIBE / BY } \\
\text { BOXPLOT / BY }\end{array}$ \\
\hline Attribute & CHISQUARED & $\begin{array}{l}\text { TWOTEST } \\
\text { ONEWAY }\end{array}$ \\
\hline
\end{tabular}

Figure 3

\section{SUMMARY}

The teaching approach outlined above has been used successfully by myself and colleagues for the last few years on first level Business Statistics courses. The material delivered is not substantially different from the material delivered on the traditional approach. The emphasis and value placed on certain concepts and ideas has changed. The formal introduction of probability theory is questioned at the introductory stage is seriously questioned. No formal probability theory is discussed, intuitive ideas of the distribution of a variable are described with the key ideas of describing the structure of the distribution in terms of its centre, width and shape are developed.

The main advantage to the student is the provision of a consistent framework for data analysis. The need for a theoretical framework is clear and the better students are enquiring about the more formal approaches. A first course of this type seems to provide a framework for a more rigorous follow on course. The framework as outlined allows for the development of the statistical modelling ideas.

A major difficulty is obtaining data sets that are within the student's experience and contain a sufficient number of observations and variables, with a sufficient to be of value. In general the statistical journals do not provide this type of data. The market research areas tend to have confidentiality problems. The data sets used within these courses are simulated data sets. To construct a realistic data set to meet the requirements of this type of course is difficult. 


\section{REFERENCES}

Chatfield, C. (1991) Problem Solving A Statistician's Guide. Chapman \& Hall, London. Ryan, B. F., Joiner, B. L. and Ryan, T. A. Jr (1985) Minitab Handbook, 2nd edn., PWS-Kent, Boston

Velleman, P.F. and Hoaglin, D. C. (1981) Applications, Basics and Computing of Exploratory Data Analysis, Duxbury Press, Boston, Mass.

\section{BIOGRAPHY}

George Rawlings is currently Principal Lecturer in Management Sciences at the University of Central Lancashire. After working in various industrial positions he joined the academic staff at Newcastle Polytechnic. With a group of staff developed the Business Information Technology courses and was the first course leader for the Post-Graduate Diploma in BIT. Moving in 1987 to his current post with the responsibility of developing BIT courses at UCL.

With a strong teaching team has key interests in teaching a range of quantitative subjects through IT. 